\title{
Application of PLC technology in electrical engineering and automation control
}

\author{
Luxian Fang \\ The College of Post and Telecommunication of Wuhan Institute of Technology, Wuhan Hubei, \\ 430073, China
}

Keywords: PLC technology, Electrical engineering, Automation control, Application.

\begin{abstract}
Programmable logic controller, i.e. PLC, as a kind of reservoir with the programmable function can be effectively used for storage of interior program and can conduct each operations according to the instructions. Inside electrical engineering and automation control and other special software systems, PLC has played important role with its high-efficiency technology and accurate executive capacity. In recent year, China has been actively engaged in modernization construction with electrical engineering accelerating. Under this circumstance, it is of great significance to strengthen the research on the application of PLC technology in electrical engineering and automation control.
\end{abstract}

\section{Preface}

The sufficient application of electrical engineering and its automation control is based on the background of information technology high-speed development which has greatly promoted the progress of electrical engineering and its automation control. Currently electrical engineering is with highly automation level and reliability has become an important characteristic of system operation. Based on this, the article firstly makes a brief introduction to PLC, discusses the advantages of application of PLC technology in electrical engineering and automation control, and on the basis of further research on the practical application of application of PLC technology in electrical engineering and automation control analyzes some problems to pay attention and the future development trend in order to lay a theoretical foundation for the development of relevant field.

\section{Introduction to PLC}

Programmable Logic Controller, i.e. PLC, is the outcome combining the functions of logic controller and reservoir, and one of major characteristic in PLC invocation procedure. PLC will be completed inside the reservoir during the process of expanding program storage. Users must input orders into it for application, and PLC will make a detailed analysis on the instruction combining and applying interior practical control, arithmetical operation and logic calculation and other functions to accurately perform tasks on this basis ${ }^{[1]}$. It is exactly because of its functions mentioned above that during controlling different kinds of mechanic equipment operators must input various numbers and simulation analogs. In this way, related equipment can implement various production tasks based on program design and instructions.

PLC is actually a special computer in essence capable of controlling various programs in industrial productions. Like computers, PLC contains hardware components such as communication modules, technological orientation, reservoir, CPU, and power supply.

Based on specific instructions, PLC proceeds three steps during the implementation operation: firstly input sampling, and implement user program and at last output refresh. A scanning cycle can 
be completed in above three steps, and then PLC needs to make repeated scanning of performing instructions. Regarding the application of PLC technology in current mechanical production, DCS and FCS are key points for PLC controlling functions to be fully exerted.

\section{Advantages of application of PLC technology in electrical engineering automation control}

PLC's technical features when effectively combined with information technology is the key to promote the rapid development of electrical engineering automation. In current stage, electrical equipment has higher demand for calculation speed and information storage volume. The effective application of PLC technology can satisfy the operation demand of modern electrical engineering automation because R\&D and application of PLC technology is closely related with people's life and industrial production so that it has strong practicability. The application of PLC technology in mechanic equipment can build a more harmonious environment laying solid foundation for strengthening man-machine interaction. Because of this, feasibility, comprehensiveness and practicability can be seen as the major features of PLC technology to lay solid foundation for better and faster development of electrical engineering automation. More importantly, PLC has been widely applied to other fields. Higher intelligence level, larger integration density and faster operation speed are characteristics PLC technology has displayed in nowadays application which conform to the demand for electrical engineering automation and development. Meanwhile, PLC has strong proactivity and integration available to effectively integrate with other technologies so as to lay solid foundation for optimizing electrical engineering automation ${ }^{[2]}$.

\section{Practical application of PLC technology in electrical engineering automation control}

When PLC technology appeared at first, its functions and values could not be fully presented in electrical engineering automation control. Traditional electrical engineering automation control has single functions. With the rapid development of industrial production and electrical engineering field, electrical engineering automation control has been asked for higher demand. In this case, PLC technology has played its full function in electrical engineering automation control, the practical application of which mainly reflected in sequential control and switching value control.

\section{Application in sequential control}

In recent years, with the rapid development of social economy, enterprise need to face up to more fierce market competition in long-term development. In this case, to improve their comprehensive competitiveness, most enterprises start to increase the application of PLC technology in production. Thus PLC technology has been applied as sequential controller for a long time. For example, thermal power plant usually produces a lot of slags and fly ashes during operation which will lead to serious environmental pollution if not cleaned up timely. To strengthen the cleaning and effectively protect environment, sequential controller has been applied effectively ${ }^{[3]}$. PLC technology contained in this device can control related equipment to timely clean slags and fly ashes based on the boiler operation condition without influencing the normal operation of thermal power plant to effectively avoid the diffusion of slags and fly ashes and improve environmental protection. It is noticeable that during the application of sequential controller, it is necessary to pay attention to designing automation system in scientific and reasonable way. Usually the system can be divided into three components as below: firstly the field sensor part can carefully record the equipment operation condition and surrounding environment parameters through detection head and transmit to the master controller so as to lay solid foundation for operators to reasonably send control instructions; secondly remote control part. The normal operation of this part can greatly contribute to the realization of remote operation, improving working environment and efficiency. Meanwhile, it can help reduce human resource costs during the company operation. The last one is main station layer. Its effective operation can guarantee that the sequential control function of PLC technology can be fully played, so that it is the application core of sequential control. 


\section{Application in switching value control}

The important value of applying PLC in electrical engineering control is mainly reflected in: as reservoir can be programed, so that it can be cooperated with virtual relay in a more scientific way. Based on this, it is possible to make reasonable judgement on the performance and quality of virtual relay; when on-off control is applied in relay, it requires for a long time for relay to react. Thus, effective control cannot be achieved in short-circuit protection. It can be seen that, in long-term practical application, the defects of PLC technology have exposed so that during its application in different production fields, it requires to take specific measures based on practical demand to improve the function and applicability of PLC technology ${ }^{[4]}$. During the process, if automatic switchover system needs to be applied in production, it must be effectively integrated with PLC technology so as to make up for the defects of relay of long-time reaction to on-off control, and to lay foundation for improving the operation efficiency of the whole system. It can be seen from this that PLC's application in the switching value has produced great effectiveness.

\section{Problems of applying PLC technology in electrical engineering automation control}

In recent years, under the background that information technology advancement, intelligence has become the inevitable trend of electrical engineering automation control development. The effective application of PLC technology can make it happen because during combining IT with electrical engineering automation control and improving PLC technological theories, the value of PLC in electrical engineering automation control has stand out. In current stage, China must intensify the strength in R\&D and application of PLC technology for promoting the complete reform in electrical engineering automation control field. During this process, below problems need to be noticed:

Firstly, the in-depth development of PLC technology must be established on the basis of satisfying the demand for electrical engineering automation control development. The country and related fields must enlarge capital investment and provide research and practice platform of PLC for research staffs to promote its in-depth development based on masses of practice, and promote the close integration of PLC technology and electrical engineering automation control so as to establish correct direction of PLC development and application and guide electrical engineering automation control to further develop.

Secondly, strengthen talent cultivation. During improving the development of electrical engineering automation control system, talent who have strong professional knowledge, capacity and innovation is the key. Under this case, enterprises should provide employees with platforms for building their professional ability and guide employee to participate related training through incentive mechanism, so as to help them to master in-depth PLC technology and apply to practical work. Guide practice with theories and realize theoretical innovation in practice to lay solid foundation for satisfying the demand of electrical engineering automation control system design.

Thirdly, intensify the construction of related system and standard so as to know effective ways of PLC application into electrical engineering automation control. Promote the application and development of PLC technology and form unified standard in the industry to lay good foundation for China's electrical engineering automation control intelligence development, and meanwhile, effectively scandalize the application behaviors of PLC technology ${ }^{[5]}$. For example, apply PLC technology into electrical engineering automation control through sound communication mechanism and promote the communication between system utilization units with design units so as to keep perfecting system design from the practice and solve various operation problems fundamentally.

Fourthly, normally electrical engineering automation control system will be exposed into tough environment during its practical operation. For example, it is necessary to increase the application of anti- jamming equipment in severe electric wave interference environment so as to lay good foundation for PLC technology to play a useful role in electrical engineering automation control system. 


\section{Development trend of the application of PLC technology in electrical engineering automation control}

The effective application of PLC technology has greatly improved the production efficiency of electrical engineering thus it has great driving force for the progress of electrical engineering technology. New time development of electrical development has a higher demand for electrical engineering automation control, this PLC technology itself must own higher applicability to satisfy the development of electrical industry. From another point of view, it has laid solid foundation for the integration of computer technology and PLC technology. Under this case, the effective application of PLC in electrical engineering automation control will be one of important trends of the technology development. When improving functions of PLC technology, its practicability has been improved also with strong use functions reflecting into program design and product type development. In current stage, China will strengthen the automation degree of electrical engineering while intensifying R\&D and application of PLC technology.

\section{Conclusion}

To sum up, in recent years, the rapid development of science and information technology has laid solid foundation for the completion PLC functions. Now PLC technology can be widely applied to various industrial production fields, controlling mechanical equipment through instructions and computers so as to finish production tasks more effectively. The application of PLC technology in electrical engineering automation control mainly reflects two aspects: sequential control and switching value control. The achievements have attracted people's attention, but in the long term, only through strengthening the research staffs' knowledge of the values and noticeable problems in application process can help the technology be further improved.

\section{References}

[1] Li Qiang. Analysis on the application of PLC technology in Lianyungan port electrical engineering automation control. The Merchandise and Quality, 2015(24):215-215.

[2] Wang Yuzhong, Yu Fashan, Liang Chao. AC lifter's electrical system improvement based on PLC technology. Coal Science and Technology, 2015, 33(11):40-42.

[3] Weng Guoqing, Chen Mingjun, Zhang Youbing. Optimization of "modern electrical control and PLC technology" course practical procedures. Proceedings of the sixth national college electrical engineering and automation specialty teaching reform seminar, 2014:617-621.

[4] Yu Yongkang, Xi Minyun. Three-cell ultrasonic cleaning machine’s control system design based on PLC technology. Mechanical \& Electrical Technology, 2014(4):16-18.

[5] Zhao Baoyi, Wang Ning, Meng Sansan. LED lighting control system design based on WIFI and PLC technology.2013 Proceedings of laminating power and intelligent control technology seminar. 2013:257-262. 